\title{
The Study of Meta-Synthetic Hall Based on Simulation
}

\author{
Xinjun Zhao ${ }^{1, ~ a ~, ~ L i n g j i e ~ K o n g ~}{ }^{1, b}$ and Junyan Zhao ${ }^{1, c}$ \\ ${ }^{1}$ Science and Technology on Complex Land Systems Simulation Laboratory country \\ azhaoxjmail@126.com, bivyklj@163.com, czhaojy1980@sohu.com
}

Keywords: complicated large system, Meta-Synthesis methodology.

Abstract. The meta-synthetic methodology is the scientific and effective way to solve the problems of the complicated large system. In this article, we put forward a Meta-Synthesis Hall based on simulation. Through the Hall, we combined the expert, repository, and simulation test together, building an environment to realize the Meta-Synthesis methodology. We can solve the demonstration and construction problems of complicated large system in this environment.

\section{Requirement}

The complicated large system consists of many functional subsystems, which hinge and merge each other, is a highly synthesis and integration system. It is a huge and difficult work to construct and research Complex land system; there are a lot of uncertain and complicated factors. To construct the system is facing great difficulties and risks.

In order to solve the problems in the process of constructing complicated large system, and reduce developing risk, it is become more important to built a suitable demonstrate method in the developing complicated large system. The Meta-Synthesis method based on simulation is an effective demonstrate method to carry out complicated large system.

Meta-Synthesis method is the synthesis based on various integrations; the synthesis is above all kinds of integrations. Meta-Synthesis focuses on synthesis. Synthesis means broader and deeper research, reflects a qualitative change. Integration pay attention on the physical meaning mainly reflects the quantitative change. The studies of Meta-Synthesis emphasize the system of systems.

The Meta-Synthesis methods combined the expert system, information system and compute system, constitute a highly intelligent man-machine system. It can integrate people's thinking, experience, knowledge, and all kinds of intelligence, rise from qualitative to quantitative understanding.

The application of this methodology should decompose the large system under the overall guidance. After the study on the subsystems, the synthesis integrated the subsystem into the whole, reach for the purpose to solve the problem as a whole. In this point, the Meta-Synthesis method absorbs the superiority of reductionism and holism, and at the same time to make up the limitations of them, it is the combination of reductionism and holism.

Meta-Synthesis method points out the process to solving the problem of complicated large system. This process starts from the question; the question is precisely description of the problem with complicated large system. And then to put forward empirical assumptions, this step is combination of the related scientific theories, experience knowledge, expert judgment, and wisdom of expert system through discussing way, and it is often qualitative. Then, precision is strictly confirmed through the man-machine experiment, human-computer interaction, repeated comparison, to get a clear conclusion of empirical assumptions. If certain empirical assumption is right, the conclusion is scientific with the complicated large system. If empirical hypothesis is denied, you need to modify empirical hypothesis, puts forward a new empirical assumptions, repeat the above process.

\section{The architecture of Meta-Synthetic Hall based on simulation}

The Meta-Synthetic Hall is a concrete application of meta-synthetic methodology. The Meta-Synthetic Hall consists of three parts: expert system, machine system and knowledge system, the expert system and machine system is the carrier of knowledge. The three system combine high 
intelligently to form man-machine system, not only has the knowledge and information collection, storage, transmission, analysis and synthesis function, more important is has the function to generate new knowledge and intelligence.

The Meta-Synthetic Hall Based on Simulation is a special application of the meta-synthetic hall using simulation technology. The Hall was build based on the simulation test, the expert demonstration and information resources. The Hall organizes simulation system, expert group and the information repository together with the distributed interactive network, is shown in Fig 1. The Hall form a kind of high level matrix system, vertical hierarchy, horizontal distribution and interaction each other. The Meta-Synthetic Hall Based on Simulation provides a standardized, structured form to solve the problem of the complicated large system.

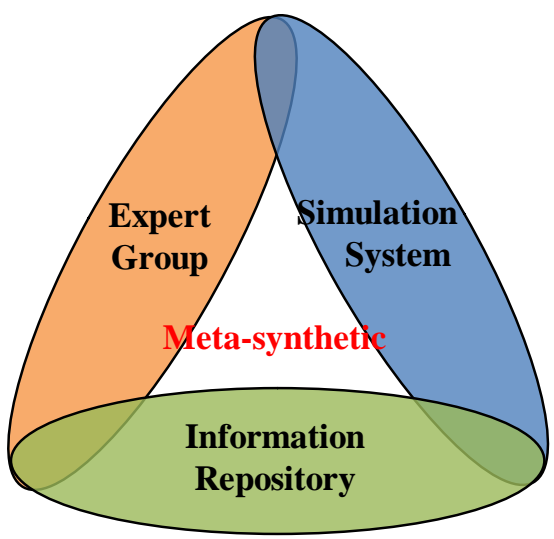

Fig 1. Meta-Synthetic Hall Based on Simulation

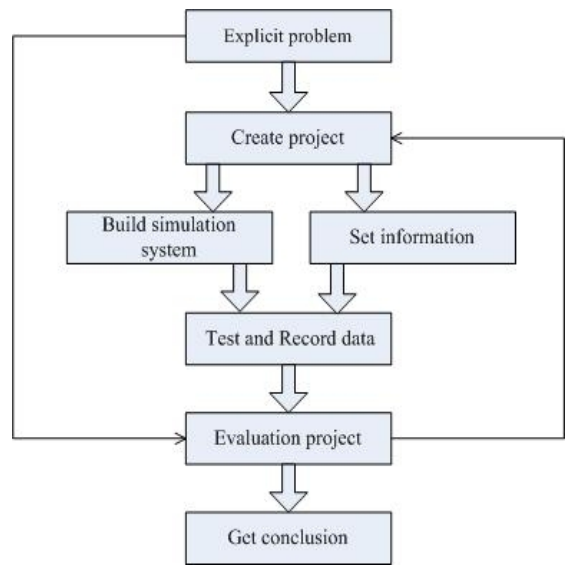

Fig 2. Meta-Synthetic Hall Process

The Expert Group is core of the Hall, was constituted with the persons from all kinds of related field. They not only give the question that we met, but also put forward the project to solving the question. Of course, the project is the hypothesis first, but in the end it will become the true mostly. The Expert Group is the intelligent generate place, it cannot be replace by any machine. With the help of simulation test and repository information, The Expert Group can adjust and optimize the project to meet requirement of the question.

The Simulation System is the parallel system of complicated large system (it may be exist or may be not) with simulation technology. The simulation system has same characters with the complicated huge system and can replace it to some extent. It is serial and has different accuracy level; it may be simple and may be complex to satisfy the requirement of the research. The most important function of the Simulation System is the test on it. The Simulation System can run all kinds of tests that were give out to verify the project of the Expert Group, and generate the data to be analysis by experts.

The Information Repository is a huge database, it store and manage all the data of the complicated large system. The expert and simulation test can get all the information they need from the Information Repository and put the new information into it also. How to organize the huge information with high efficiency is the big problem the Information Repository will meet.

In order to study the complicated large system, we need to do a series science and experience activities. The Meta-Synthetic Hall Based on Simulation follows the scientific and experiment integration means, combining intelligence and knowledge. The Hall has the ability to study and solve the problem of the complicated large system.

The Meta-Synthetic Hall Based on Simulation is an autonomous system and operates with the definite process, is shown in Fig 2, generally the process have five phases. First, we should give the problem related with the complicated large system to the Hall, experts of the Hall need explicit the problems in order to analysis it. Then, the Expert Group will study the complicated large system to get the project that should solve the problems. Third, the Hall should build the simulation system of the 
complicated large system and put the information related with the system into the repository. Fourth, the Hall carries out the tests according with the requirement that study need and record the test data into repository. Fifth, the experts analysis the data in repository and evaluate the efficiency of project. If through the experiment we can prove the project can solve the problems, the Hall will get the conclusion and give out the project to solve the problem of the complicated large system, otherwise, the Hall will began the other circle at second phase. Generally, the Hall should circle the process many times to get the conclusion, and in the process the Hall need to interchange information with other system frequently. So, the Meta-Synthetic Hall itself is an open, dynamic system, continuous development and evolution.

\section{The simulation test system}

In order to support the complicated large system research and analysis, we need to build a large-scale simulation system. The simulation system is the parallel system of the actual system, they are similarity, but different actually. The simulation system can be construct, virtual, or reality system to be built with the simulation technology. It have the same component, logistic, characteristic with the actual one, but it is simply and can be destroy and rebuilt repeatedly many time easily. Because we cannot test with the actual complicated large system frequently, for example the society, the war, the environment, so we need to build the simulation system to study the actual complicated system.

For the different research purpose, we can build different simulation system to satisfy the different requirement. One complicated large system can have many simulation systems, some are complex, and the other may be simple. Simple or complex is not important point; the important is that the simulation system can replace the actual one to some extent and at some field, so the test at the simulation system should be same with the actual system. Generally speaking, the simulation of complicated large system is a huge and difficult work, and the simulation system is also complicated. We need to spend more time and money on it to build a parallel simulation system; it is comprehensive and accuracy enough with the research on the system.

For example, with the efficiency research of the complex land system, we build a simulation system to launch test. The simulation system of the complex land system include: environment simulation system, computer generate force system, man in the loop control system, information display system, and the simulation control and other related systems, is shown in Fig 3.

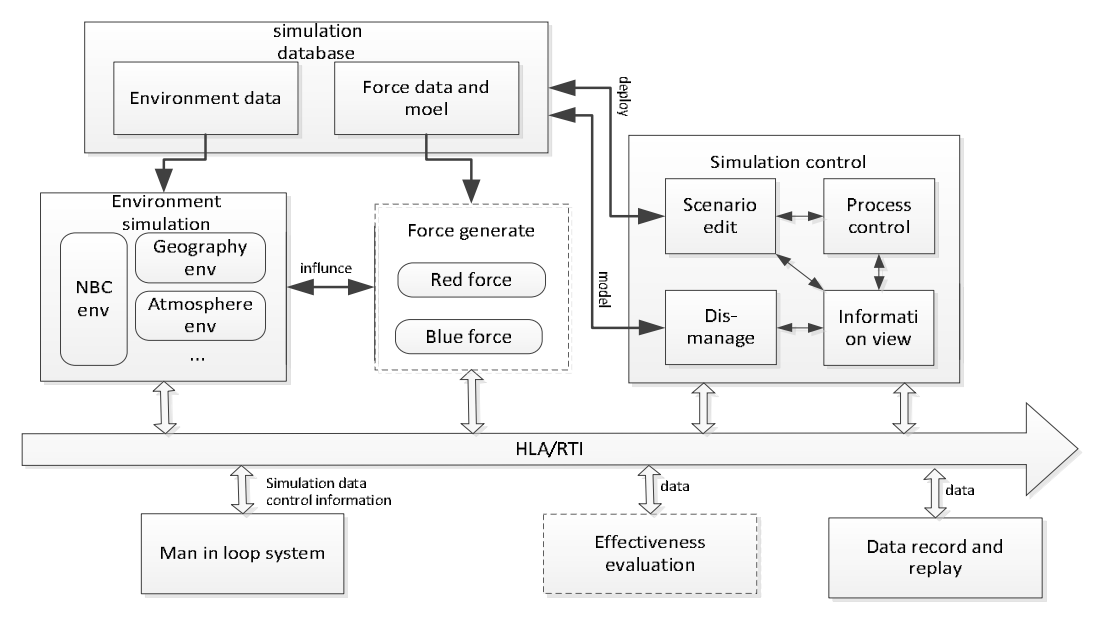

Fig 3. The Simulation System Architecture

Computer Generate Force System is the subsystem of simulation system to generating simulation entities of combat force. The simulation entities have the ability to decision and activity, simulate all kinds of force unites, including fighting unit, command unit, communication unit, and detection unit, ensure unit, etc. they can simulate weapon ability, operation activity, information transmission, 
equipment and logistics insurances, etc. The Computer Generate Force System reflected combat effectiveness of the complex land system.

Man in the Loop system is the subsystem of simulation system to give the platform which put the human being into the simulation system, lead in the perception, analysis, judgment, decision, and executive ability of the person. The person in the simulation not only input signals to the system, but also sensing variety output signal of the system. Man can make decisions according to the variation of system output signal, and feedback at the same time.

The Environment Simulation System is responsible for generating land, sea, and air environment for the simulation test. The system was able to provide dynamic simulation of battlefield environment, produce the real-time data of environment, and meet the requirements of complex land system simulation for the dynamic environment data change. The forces simulated by the Compute Generated Force System have to run under the constraint of simulation environment. The system was able to objectively and accurately reflect the environment effects on the force unities operation.

The Information Display System will visualize the information of simulation test in the process. The simulation process can produce all kinds of data, and the amount of the data is huge. How to see and realize the huge data is a big problem, the graphical of the data is the efficiency method to solve it. Through different kinds of information display way, the Information Display System cans convenience the scientific person to analysis and study of simulation test data.

The simulation system has other kinds of subsystem to maintain the simulation run smoothly, including simulate control system, record system, playback system. Simulation control system is responsible to control the simulation process, record system can record simulation data completely, and playback system can run simulation process once more with the simulation data.

\section{The expert panel}

Application meta-synthetic method need to add intelligence of experts into the complicated large system research work, so we should establish an expert group. Meta-Synthetic Hall is used to study of complicated large system; the expert group is the key to implement this methodology mechanism. The expert group will analysis problem of the complicated large system and give out the method to solve the problem. The expert group does what the machine cannot do, so they cannot be replace by machine system.

From the application requirement, the expert group composed of the experts in all aspects of the system, including requirement, demonstration, design, manufacture, application, testing, and evaluation. The leader of experts has more broad range of knowledge, he must know all the aspect to the complicated large system, and can see the large system from the higher level than other experts. The else expert will do the work belongs his field, and interacted each other. Based his work, the expert should give result and put forward advice to the leader.

Circle Improve

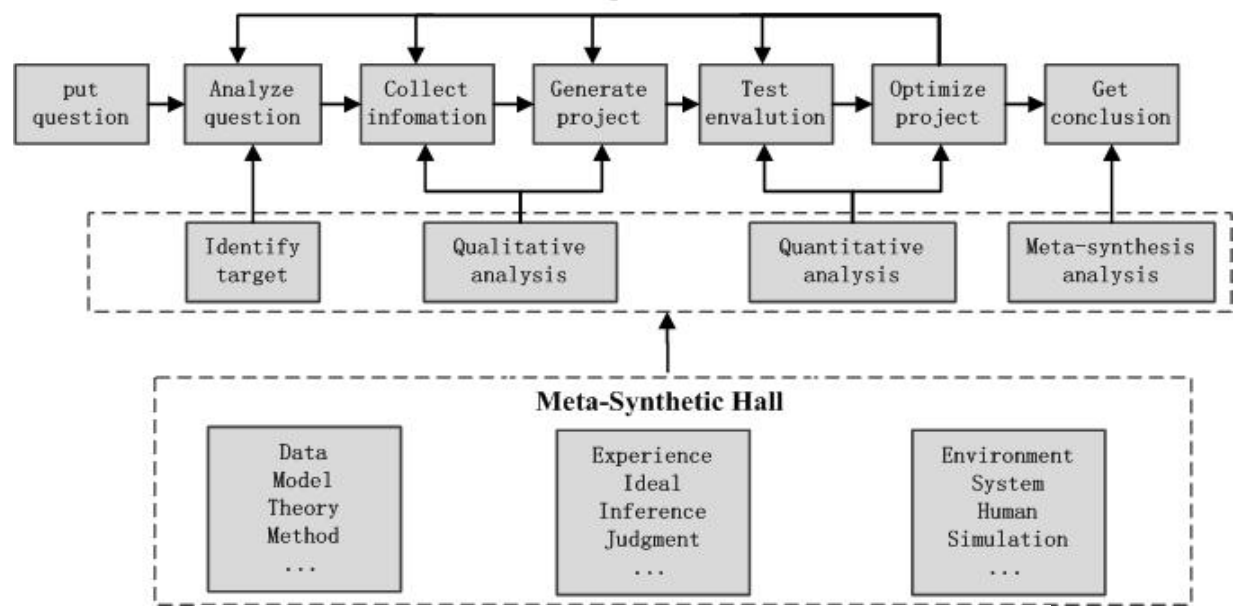

Fig 4. The expert panel deliberates procedure 
Applying meta-synthetic method to study the system overall, the expert panel design overall plan and implementation way of system. Experts in the group are working together tightly, different from traditional individual way in the scientific research. The research mode is important; the experts need have a unified platform and a study mechanism. The unified platform was build based on repository and simulation system, give experts kind's tools to realize his ideal and interchange it with other expert easily. The study mechanism confirm the phases that should solve different problems during the research process, is shown in Fig 4, the experts should accomplish the assigned task in the different phase.

The discussion is important in the expert group, it is also important in the Meta-Synthetic Hall, it is the most useful pattern to put out a new ideal and make it more and more correct. But in the discussion, there is a problem that the different point of view in the experts will often contradict. To solve this problem, we need have a consensus mechanism; otherwise we will discuss all days and will have nothing at all. The consensus mechanism gives out a program to make the experts discussion plenarily and optimize the result in the end, draw up the rules that can ensure the expert express view freely and avoid someone impose his view on others.

The expert group's important aim is to look the system from high level; any system is a part of the larger system which it belongs to. First of all, the experts must see the system from the point to realize the larger system and figure out the relation between the system and the larger one. And then, the expert analysis the system itself and design subsystems from whole system. The requirement of each subsystem is first proposed from the standpoint of the implementation of the whole system. The relationships between the subsystem and system, and between the subsystem each other, need to consider first from the system overall. Then, they can analysis the different part of system respectively, but they must realize all the time that any part of system is not lonely. In briefly, put the overall solution with the complicated larger system is scientific, feasibility and operability process for the expert group.

\section{The repository}

The repository is store and manages specific knowledge related to the complicated large system, it is update system, renew the old data and input new data constantly. The information stored in the repository is renovated by the experts and test system without stop. The content in the repository is classified and managed scientifically, mainly includes: system requirement, purpose, composition, test, analysis, technical documentation, and so on.

Repository content covers for test, analysis and evaluation. It has various relevant information for complex land system, contains a large amount of basic data and model for computer generated forces, includes both military scenario data and text for simulation, 3D terrain, entity model, battlefield environment information for combat simulation, large amounts of data from the various simulation test, and evaluation results of the data, and so on. It contains the methods and models used for analysis and evaluation, etc. and also include other resources related to the simulation for the research and development of the system.

All experts put the information about their work into the repository according the classification. At the same time, they can see the information of the other expert easily without barriers. So the repository can accumulate the knowledge not only from one person, but also from all the experts of the group. Through the interchange the information about their ideal, the Hall can greatly accelerate the emerging of new ideal.

The repository also stores all the data that should be prepared for the simulation system; it is a hard work for complicated large system to prepare lots of data for simulation. The simulation system need read all the data at the beginning of simulate, and then the data will drive simulation process and generate result data. The result data was put into the repository also can be finding out by the experts. 


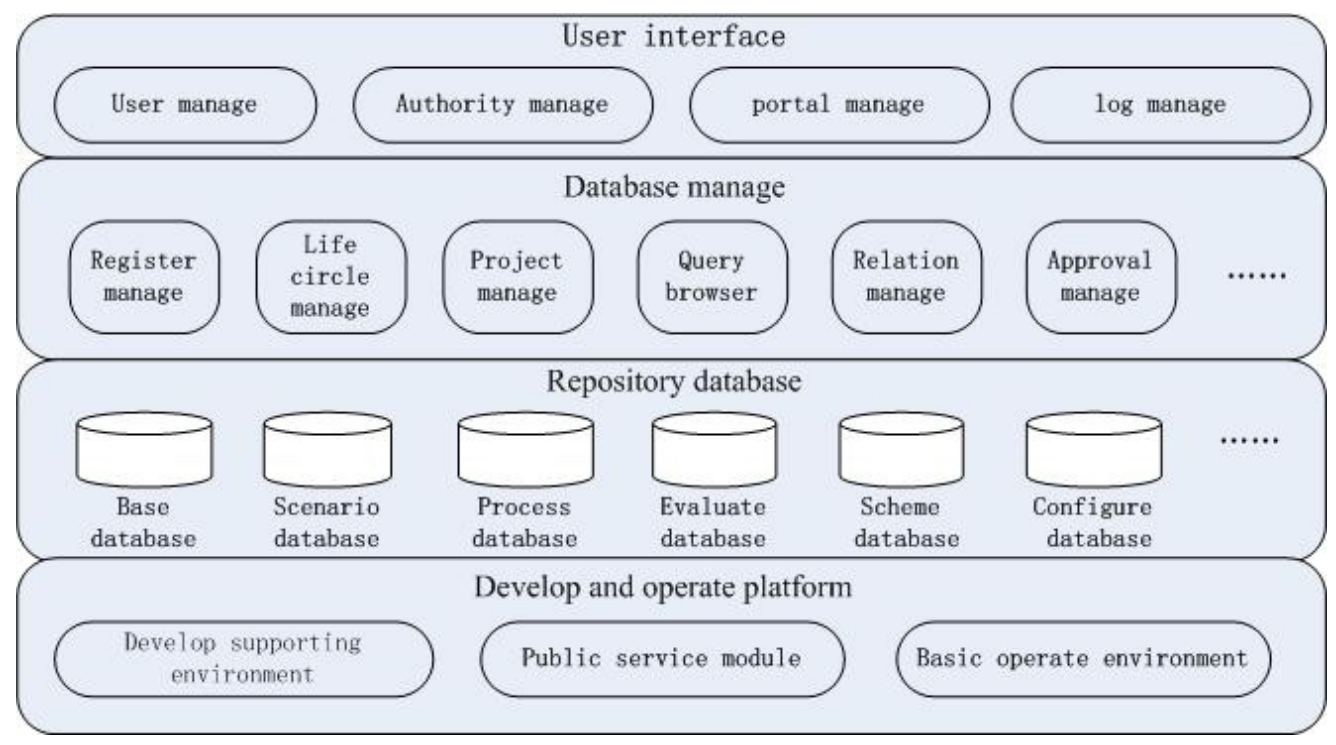

Fig 5. The repository layer construct

Using four layers system structure, is shown in Fig 5, the repository build resources environment and have management capabilities of simulation data and the model, supporting high speed access and query the data and model from the resources. It realized the dynamic query, search, browse, upload, download, can accomplish data integrity and consistency, and other functions.

\section{The conclusion}

The complex land system is an open complicated giant system, so the system research and development need more scientific systems engineering methodology. The meta-synthetic is the scientific methodology to solve giant complex system problem. Through combining expert panel, simulation system and repository, we can build Meta-Synthetic Hall. The Meta-Synthetic Hall based on simulation is the scientific and effective way to solve the problems of the complex land system demonstration and construction.

\section{References}

[1] Distributed Simulation Systems Specification, Version 2.0, OMG, Inc, 2002.11.

[2] C.L.Kang, National Defense System Analysis Methods, In Chinese, National Defense Industry press 2003.2.

[3] Ralph Liebert, Michael J. O'Connor, Richard Wyman, Technical Aspects of the Multi-Service Distributed Environment to Support Joint Test and Evaluation, Simulation Interoperability Workshop Sping Conference, 2006 Spring.

[4] D. C. Sun, F. Y. Lin, Systems engineering methodology, In Chinese, Tinghua university press, 2005.08

[5] Craig T. Doescher, Randy Kubik, Michael Wilmer, Is It Live or Is It Memorex: The Power of Live/ Sim Integration, Simulation Interoperability Workshop Sping Conference, 2003

[6] Dirk Brade, Conceptual Modeling Meets Formal Specification, Simulation Interoperability Workshop Sping Conference, 2003. 